\title{
The Theoretical Frequency of Classical Nova Outbursts as a Function of White Dwarf Mass
}

\author{
Michael Politano(1), Mario Livio ${ }^{(2)}$, James W. Truran $^{(3)}$ and Ronald F. Webbink ${ }^{(3)}$ \\ ${ }^{(1)}$ Department of Physics, Arizona State University, U.S.A. \\ ${ }^{(2)}$ Department of Physics, Technion, Haifa, ISRAEL \\ ${ }^{(3)}$ Department of Astronomy, University of Illinois, U.S.A.
}

\section{INTRODUCTION}

Using the distribution of white dwarf masses in zero-age cataclysmic variables (CVs) calculated by Politano and Webbink (these proceedings), the relative frequency of classical nova outbursts as a function of white dwarf mass is calculated. These results are compared with the results obtained by Truran and Livio (1986), who calculated the same function, but used a distribution of white dwarf masses in CVs calculated from a Salpeter initial mass function and a particular progenitor mass-white dwarf mass relationship for single stars.

\section{OUTLINE OF METHOD}

We wish to calculate the frequency of classical nova outbursts as a function of white dwarf mass. To do this, we require two quantities: 1 ) the recurrence frequency of classical nova outbursts (i.e., how many outbursts per year) and 2) the white dwarf mass spectrum in classical novae (i.e., number of novae per white dwarf mass). We discuss each of these in turn.

\section{Recurrence frequency}

Truran and Livio (1986) have shown that the recurrence frequency of outbursts of classical novae may be estimated by:

$$
\nu_{\text {rec }}=\left(G \dot{M} / 4 \pi P_{\text {crit }}\right)\left(M_{W D} / R_{W D}^{4}\right)
$$

where $\dot{M}$ is the rate of accretion of matter by the white dwarf, $P_{\text {crit }}$ is the critical pressure at the base of the accreted envelope necessary to achieve a thermonuclear runaway, and $M_{W D}$ and $R_{W D}$ are the mass and radius, respectively, of the white dwarf. The radius of the white dwarf may be written as (Eggleton 1982):

$$
R_{W D}=8.5 \times 10^{8}\left[1.286\left(M_{W D} / M_{\odot}\right)^{-2 / 3}-0.777\left(M_{W D} / M_{\odot}\right)^{2 / 3}\right]^{1 / 2} \mathrm{~cm} .
$$

Assuming a constant accretion rate, as in Truran and Livio (1986), and a value of the critical pressure, $P_{\text {crit }}=2 \times 10^{19} \mathrm{dyne} / \mathrm{cm}^{2}$, as implied by numerical calculations (see again, Truran and Livio [1986]), make the expression for the recurrence frequency of white dwarfs (equation 1) a function only of white dwarf mass.

\section{White dwarf mass spectrum}

Lacking any detailed knowledge of the mass spectrum of white dwarfs in classical novae, Truran and Livio (1986) used a progenitor mass-white dwarf mass relation for single stars and a Salpeter initial mass function for the progenitor primaires to approximate the white dwarf mass spectrum in CVs. Since then, work has been done by Politano and Webbink $(1988,1989)$ which, for the first time, provides a theoretical distribution of the white dwarf masses in "zero-age", or newly-forming, CVs. A summary of the details of the calculation is given in Politano and Webbink (1988). Under the assumptions that the mass of the white dwarf remains essentially unchanged as a result of the secular evolution of the CV and that the distribution of white dwarf 
masses in classical novae may be taken as a direct, similar subset of the distribution of white dwarf masses in CVs, we have used their distribution for our white dwarf mass spectrum.

\section{RESULTS}

The results of our calculations are shown in Table 1. $M_{W D}$ is the white dwarf mass in solar masses, $f\left(M_{W D}\right)_{T L}$ is the outburst frequency calculated by Truran and Livio (1986) and $f\left(M_{W D}\right)_{P L T W}$ is the outburst frequency calculated using the white dwarf mass spectrum calculated by Politano and Webbink (1989). The outburst frequencies listed in Table 1 have been normalized, so that the total outburst frequency integrated over the entire range of white dwarf masses in the table is equal to 1 .

Therefore, the entries in columns 2 and 3 represent the fractions of all outbursting systems in the given white dwarf mass range ( 0.1 solar mass) centered on the mass in column 1 (except for the last entry, which is the fraction of outbursting systems in the mass range 1.35 to 1.4 solar masses).

\section{Table 1}

Outburst Frequencies as a Function of White Dwarf Mass

\begin{tabular}{|c|c|c|}
\hline$M_{w d}$ & $f\left(M_{w d}\right)_{T L}$ & $f\left(M_{w d}\right)_{P L T W}$ \\
\hline 0.60 & 0.103 & 0.035 \\
\hline 0.70 & 0.053 & 0.087 \\
\hline 0.80 & 0.042 & 0.076 \\
\hline 0.90 & 0.040 & 0.060 \\
\hline 1.00 & 0.046 & 0.058 \\
\hline 1.10 & 0.062 & 0.069 \\
\hline 1.20 & 0.100 & 0.098 \\
\hline 1.30 & 0.232 & 0.212 \\
\hline 1.35 & 0.322 & 0.306 \\
\hline
\end{tabular}

\section{CONCLUSIONS}

1. The relative outburst frequencies in classical novae calulated using the white dwarf mass spectrum of Politano and Webbink (these proceedings) agree with those calculated by Truran and Livio (1986) for white dwarf masses greater than approximately 1.0 solar mass.

2. The average white dwarf mass in outbursting nova systems, based on these calculations, is 1.14 solar masses.

3. Approximately one-third of all outbursting systems have a mass of 1.35 solar masses or greater and thus should probably be $\mathrm{O}-\mathrm{Ne}-\mathrm{Mg}$ white dwarfs. This is consistent with observed $\mathrm{O}-\mathrm{Ne}-\mathrm{Mg}$ novae.

4. Due to 2.) and 3.) above, the white dwarfs in oubursting nova systems cannot be regarded as typical for CVs in general, and, even more so, these white dwarfs are not good representatives of the general white dwarf population.

\section{ACKNOWLEDGEMENTS}

The authors wish to express their thanks to Professor Dr. Kippenhahn for the hospitality of the Max-Planck-Institut für Astrophysik, Garching bei München, where their work on this paper was completed.

\section{REFERENCES}

Eggleton, P. 1982, private communication.

Politano, M. and Webbink, R.F. 1988, in IAU Colloquium 114, White Dwarfs, ed. G. Wegner, (Springer-Verlag: Berlin), p.440.

Truran, J.W. and Livio, M. 1986, Ap.J., 308, 721. 